\title{
A Missense Variant (R239Q) in CCN3 Induces Aberrant Apoptosis in the Developing Mouse Brain
}

\author{
Hyunduk Kim ${ }^{1,2,}$, Hayoung Yang ${ }^{1,}$, Dong Kyun Woo ${ }^{3}$, \\ Sung-Wuk Jang ${ }^{2, \dagger}$ and Sungbo Shim ${ }^{1, \uparrow}$ \\ ${ }^{\text {I} D e p a r t m e n t ~ o f ~ B i o c h e m i s t r y, ~ C h u n g b u k ~ N a t i o n a l ~ U n i v e r s i t y, ~ C h e o n g j u ~ 28644, ~ K o r e a ~}$ \\ ${ }^{2}$ Department of Biomedical Sciences, University of Ulsan College of Medicine, Seoul 05505, Korea \\ ${ }^{3}$ College of Pharmacy and Research Institute of Pharmaceutical Sciences, \\ Gyeongsang National University, Gyeongnam 52828, Korea
}

CCN3 (also known as NOV, Nephroblastoma overexpressed) proteins are involved in various pathologies during different developmental stages. We have previously shown that intracellular levels and normal extracellular secretion of $\mathrm{CCN} 3$ are important for neuronal differentiation. Furthermore, we demonstrated that a single amino acid in the CCN3 TSP-1 domain is important for extracellular secretion and that palmitoylation of CCN3 is required in this process. However, the effect of abnormal CCN3 accumulation on cells remains to be studied. Here, we found mutations in the TSP-1 domain of $\mathrm{CCN} 3$ that led to intracellular accumulation and abnormal aggregation of $\mathrm{CCN} 3$. It was observed that this mutation resulted in a phenomenon similar to neurodegeneration when overexpressed in the developing mouse cortex. This mutation also confirmed the activation of apoptotic gene expression in Neuro2a cells. In addition, we confirmed the in vivo transcriptional changes induced by this mutation using microarray analysis. We observed a significant increase in the expression of Anp32a, an apoptosis-related gene. Collectively, these results indicate that a single mutation in CCN3 can lead to abnormal cell death if it shows intracellular accumulation and abnormal aggregation.

Key Words: Point mutation, CCN3, Aggregation, Apoptosis, Neuron

\section{INTRODUCTION}

The CCN family, consisting of six members, commonly contains an insulin-like growth factor binding protein (IGFBP), von Willebrand factor type $\mathrm{C}$ repeat (VWC), thrombospondin type 1 repeat (TSP-1), and a cysteine knot motif carboxyl-terminal (CT) domain. CCN5 is an exception in that it lacks CT domains (Holbourn et al., 2008; Malik et al.,
2015). The CCN family is secreted outside the cell and is involved in various biological functions such as development, cell fate, angiogenesis, cell migration, cell adhesion, apoptosis, and cell survival (Chen et al., 2009).

In fibroblasts, CCN1, CCN2, and CCN3 can induce apoptosis as cell adhesion substrates. In particular, CCN1 binds to $\alpha 6 \beta 1$ and syndecan-4, and induces activation of Bax through p53, leading to apoptosis (Chen et al., 2009; Ren et al., 2014). On the other hand, binding of CCNs to endothelial

*Received: May 29, 2018 / Revised: June 3, 2018 / Accepted: June 4, 2018

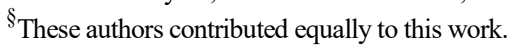

${ }^{\dagger}$ Corresponding author: Sungbo Shim. Department of Biochemistry, Chungbuk National University, Cheongju 28644, Korea.

Tel: +82-43-261-2318, Fax:+82-43-267-2306, e-mail: sungbo@cbnu.ac.kr

${ }^{\dagger}$ Corresponding author: Sung-Wuk Jang. Department of Biomedical Sciences, University of Ulsan College of Medicine, Seoul 05505, Korea.

Tel: +82-2-3010-2088, Fax:+82-2-3010-2088, e-mail: swjang@amc.seoul.kr

(C) The Korean Society for Biomedical Laboratory Sciences. All rights reserved.

(C) This is an Open Access article distributed under the terms of the Creative Commons Attribution Non-Commercial License (http://creativecommons.org/licenses/by-nc/3.0/) which permits unrestricted non-commercial use, distribution, and reproduction in any medium, provided the original work is properly cited. 
cells protects against apoptosis (Ren et al., 2014; Jun and Lau, 2011).

The Ccn3 gene was first documented in avian nephroblastoma. Its expression and activity is highly correlated with cancer and neuronal differentiation (Brigstock, 1999; Holbourn et al., 2008; Park et al., 2015). Moreover, truncated CCN3 protein is increased in human cancer cells (Perbal, 2009). In particular, CCN3 promotes cell adhesion and migration of rhabdomyosarcoma (RMS) (Zhang and Wang, 2011). Overexpression of CCN3 promotes the expression of RAB25 in neurons, thereby inhibiting neuronal outgrowth (Park et al., 2015). Despite these various intracellular effects, little is known about the intracellular function of $\mathrm{CCN} 3$ compared to its extracellular function. In addition, although each domain of CCN3 is involved in various biological processes, there is a paucity of information on the effect of a single amino acid mutation on the function of $\mathrm{CCN} 3$ or its effects on the cell.

Our previous study showed that a single mutation in the TSP-1 domain of CCN3 can inhibit normal CCN3 secretion. In particular, a single mutation analysis of $\mathrm{CCN} 3$ demonstrated that palmitoylation is important for the normal secretion of CCN3 (Kim et al., 2018). We demonstrate in this study that a different single mutation in TSP-1 induces abnormal aggregation of $\mathrm{CCN} 3$ as well as suppression of $\mathrm{CCN} 3$ secretion, leading to cell death.

\section{MATERIALS AND METHODS}

\section{Plasmids}

To construct mutant CCN3 expression vectors, a fulllength complementary DNA sequence encoding murine CCN3 tagged with a V5 epitope was inserted into the pCAGEN vector (Addgene, Cambridge, MA, USA) and subjected to mutagenesis using overlapping polymerase chain reaction (PCR) (Kim et al., 2018). To construct Ccn3-R239K, a 732 bp fragment was amplified with the primers 5'-ATGAGCCTCTTCCTGCGAAAGC-3' (forward) and 5'-CCGAACGATGCAGAGCTTAGTCTGTTTTAC-3' (reverse), and a $360 \mathrm{bp}$ fragment was amplified with the primers 5'GTAAAACAGACTAAGCTCTGCATCGTTCGG-3' (forward) and 5'-AATTTCTCCTCTGCTTGTCTTC-3' (reverse).
To construct $C$ cn3-R239Q, a 732 bp fragment was amplified with the primers 5'-ATGAGCCTCTTCCTGCGAAAGC-3' (forward) and 5'-CCGAACGATGCAGAGTTGAGTCTGTTTTAC-3' (reverse), and a 360 bp fragment was amplified with the primers 5'-GTAAAACAGACTCAACTCTGCATCGTTCGG-3' (forward) and 5'-AATTTCTCCTCTGCTTGTCTTC-3' (reverse). For each mutant, the two partially complementary PCR fragments were annealed and used as templates in another PCR with the primers 5'-ATGAGCCTCTTCCTGCGAAAGC-3' (forward) and 5'-AATTTCTCCTCTGCTTGTCTTC-3' (reverse). The resulting point mutants were digested with SpeI and inserted into the pCAGEN vector, including the V5 epitope.

\section{Cell culture and western blotting}

Neuro2a cells were cultured in Dulbecco's modified Eagle's medium (Gibco BRL, Grand Island, NY, USA) supplemented with 10\% fetal bovine serum (Hyclone, Logan, UT, USA), $0.5 \mathrm{mM}$ glutamine, and penicillin-streptomycin $(100 \mu \mathrm{g} / \mathrm{mL})$ at $37^{\circ} \mathrm{C}$ in an incubator containing $5 \% \mathrm{CO}_{2}$. Cells were transfected using iN-fect (iNtRON, Seongnam, South Korea). After $24 \mathrm{~h}$, cells were lysed in RIPA buffer (137 mM NaCl, 20 mM Tris-HCl, pH 8.0, 1\% NP-40, 10\% glycerol, $500 \mathrm{mM}$ orthovanadate, and $1 \mathrm{mM}$ phenylmethylsulfonyl fluoride). Total lysates were loaded onto $4 \sim 12 \%$ sodium dodecyl sulfate-polyacrylamide gel electrophoresis (SDS-PAGE) gels and transferred to polyvinylidene difluoride membranes (Bio-Rad, Hercules, CA, USA). The membranes were immunoblotted with anti-V5 antibody $(1: 3,000$, Invitrogen).

\section{In utero electroporation and immunohistochemistry}

All animal experiments were performed in accordance with the protocols approved by the Institutional Animal Care and Use Committee of Chungbuk National University. In utero electroporation was performed as previously described (Kim et al., 2018)

Immunohistochemistry was performed as previously described with several modifications (Park et al., 2015). Briefly, brains were dissected and fixed in $4 \%$ paraformaldehyde (PFA) overnight at $4{ }^{\circ} \mathrm{C}$ and sectioned using a vibratome (Leica). Slices were blocked with phosphate-buffered saline 
(PBS) containing $0.3 \%$ Triton X-100, 5\% normal donkey serum (Jackson Immunoresearch Labs, PA, USA), 1\% bovine serum albumin, $0.2 \%$ glycine (Sigma), and $0.2 \%$ lysine (Sigma). The slices were incubated with a primary antibody recognizing the pan-neuronal marker L1 cell adhesion molecule (L1-CAM) (rat, 1:300, Millipore) and green fluorescent protein (GFP; chicken, 1:3,000, Abcam, Cambridge, UK) in blocking buffer and with the appropriate secondary antibodies (Jackson Immunoresearch Labs, PA, USA). Specimens were analyzed with a confocal microscope.

\section{Real-time Quantitative PCR (qPCR)}

Total RNA was extracted from cells using the RNeasy Mini Kit (QIAGEN), and complementary DNA was prepared form DNase-treated total RNA using the HiSenScript RH(-) RT PreMix Kit (iNtRON, Seongnam, South Korea) according to the manufacturer's instructions. qPCR was performed using HotStarTaq ${ }^{\circledR}$ PreMix DNA polymerase (QIAGEN) and SYBR Green Mastermix (BioRad, Hercules, CA, USA) with the primers listed in Supplementary Table 1. Data were normalized to endogenous glyceraldehyde-3phosphate dehydrogenase (Gapdh) expression levels.

\section{Microarray analysis}

Microarray analysis as performed as previously described with several modifications (Park et al., 2015). Briefly, total RNA was isolated from freshly dissected cortical tissues which were co-transfected with Gfp and Ccn3-R239Q expression vectors using the RNeasy Plus Kit (QIAGEN). Isolation of cortical tissues was performed under fluorescence microscopy at $\mathrm{P} 0$. Whole mouse genome microarray was performed using the Affymetrix GeneChip Mouse Gene 430 (Santa Clara, CA). After the arrays were scanned, they were analyzed using Affymetrix Expression console DAVID. Criteria used to select genes were significant upregulation or downregulation ( $>1.5$ fold) with overexpression of CCN3R239Q compared to empty vector electroporated cortices.

\section{Statistical analysis}

Quantitative data are presented as the mean \pm standard deviation (SD) of results of separate experiments $(n=3)$.

\section{RESULTS}

\section{A CCN3-R239Q mutation inhibits callosal projection} via aberrant aggregation

Previous studies have shown that the TSP-1 domain of $\mathrm{CCN} 3$ is necessary for the extracellular secretion of CCN3. Furthermore, it was confirmed that palmitoylation of the Cys241 residue was required for normal secretion (Kim et al., 2018). Among the SNPs of human CCN3 newly discovered through the recent ExAC project (http://exac.broadinstitute.org/), there is a missense mutation in which $r s 773316453$ replaces the Arg242 of the TSP-1 domain with glutamine (Lek et al., 2016). This is consistent with the Arg239 amino acid of mouse CCN3 (Fig. 1A). There is no known biological or biochemical change due to the mutation.

To address the effect of $r s 773316453$ variant of human CCN3 on cells, we examined the expression of mouse CCN3 mutants in Neuro2a cells. This was obtained by replacing Arg239 with glutamine (Fig. 1A).

As a result, CCN3-R239K was normally expressed and secreted from cells, but CCN3-R239Q was not secreted from cells due to abnormal intracellular aggregation (Fig. 1B). We also observed that when expressed in the cortex of mice with the CCN3-R239Q mutation, abnormalities in callosal projection neuron formation occurred. Interestingly, the neurite outgrowth of CCN3-R239Q-overexpressing neurons was markedly reduced and demonstrated neuronal degeneration (Fig. 1C). Taken together, these results suggest that substitution of glutamine with the Arg239 of CCN3 not only inhibits extracellular secretion but also affects normal cell differentiation

\section{A CCN3-R239Q mutation induced the expression of apoptotic-related genes in Neuro2a cells}

We investigated the effect of the CCN3-R239 mutation on cells, which results abnormally aggregated CCN3. Previous studies have shown that abnormal aggregation of proteins can lead to apoptosis (de Oliveira et al., 2015). Therefore, we first examined the expression of apoptotic-related genes in Neuro2A cells overexpressing CCN3-R239Q mutants Puma, a pro-apoptotic gene, was the most highly expressed. 


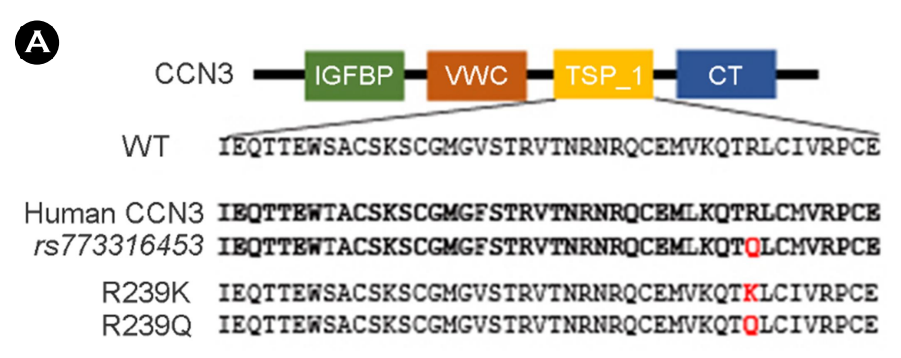

B

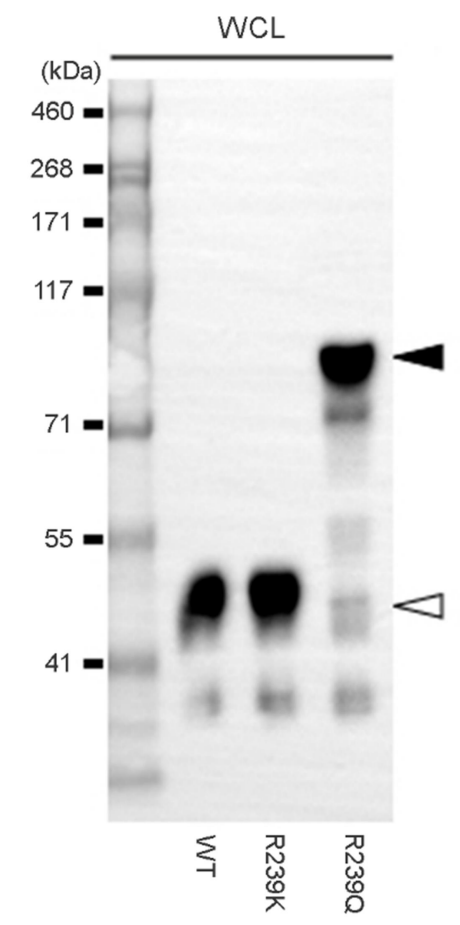

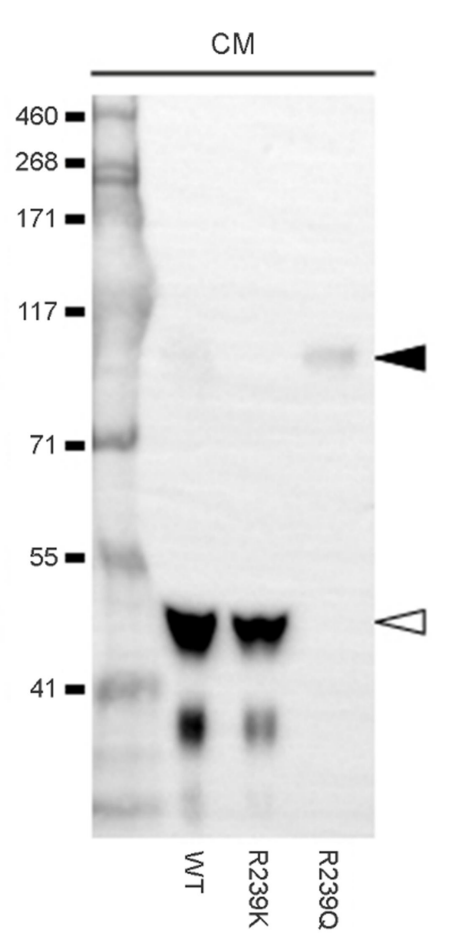

C
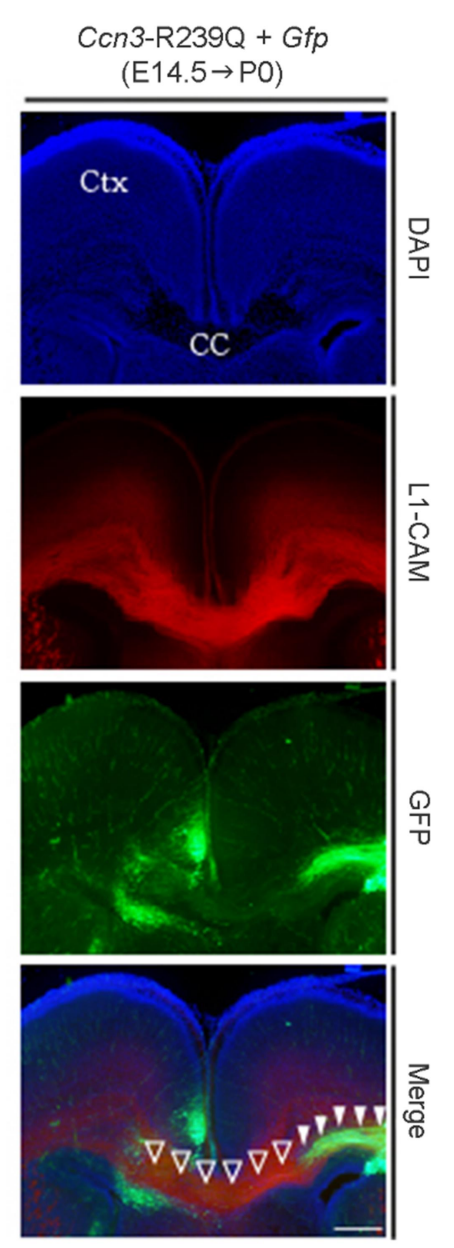

Fig. 1. R239Q mutants induced the intracellular accumulation and aggregation of CCN3. (A) Schematic of mouse CCN3-R239 mutants. (B) Western blot analysis of CCN3-R239 mutations in Neuro2a cells. The open arrowheads indicate the monomer of CCN3 and the solid arrowheads indicate the aggregated form of CCN3. WCL; Whole cell lysate, CM; Conditioned Media. (C) In vivo analysis of callosal projection formation upon expression of CCN3-R239Q mutant. In utero electroporation was performed at E14.5 with plasmids expressing the CCN3-R239Q and GFP. The open arrowheads indicate the normal paths of L1-positive callosal axons. In contrast, the solid arrowheads indicate the path of CCN3-R239Q-overexpressing neurons. Scale bar, 2 mm. Ctx; Cortex, CC; Corpus callosum.

Bcl2-like 2, an anti-apoptotic gene, was also expressed, but its levels remained at half those of Puma (Fig. 2). Although overexpression of CCN3-R239Q did not induce the expression of caspase proteins, this may have been due to the use of immortalized cell lines. These results suggest that the CCN3-R239Q mutation, when abnormally aggregated within cells, promoted the induction of apoptotic pathways rather than anti-apoptotic pathways.

\section{CCN3-R239Q mutations were sufficient to promote} apoptotic pathways via upregulation of Anp32a in vivo

Next, we investigated whether the CCN3-R239Q mutation induces the expression of certain apoptotic genes in vivo. CCN3-R239Q was overexpressed with GFP in the developing mouse cortex at E14.5. GFP-positive cortical tissue was isolated at $\mathrm{P} 0$ and transcript changes were analyzed using microarray (Fig. 3A). We observed that 48 genes (Supplementary Table 2) were increased more than 1.5 fold 


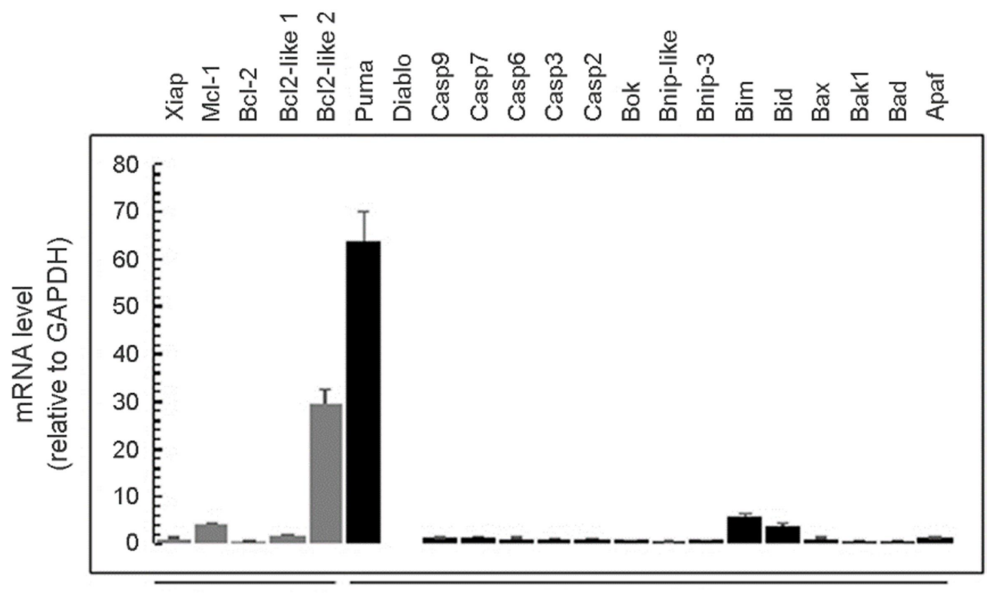

Anti-apoptotic

Pro-apoptotic
Fig. 2. Induction of apoptotic-related genes by CCN3-R239Q overexpression in Neuro2a cells. Anti-apoptotic (Xnip, Mcl-1, Bcl-2, Bcl2-like 1, and Bcl2-like 2) and pro-apoptotic (Puma, Diablo, Casp2, 3, 6, 7, 9, Bok, Bnip-like, Bim, Bid, Bax, $B a k 1, B a d$, and $A p a f)$ mRNA levels were evaluated by real time qPCR in Neuro2a cells transfected with or without CCN3-R239Q mutants for $24 \mathrm{~h}$. The relative mRNA expression levels were normalized to those of Gapdh. Results are presented as means \pm standard deviation of 3 separate experiments.
(A)

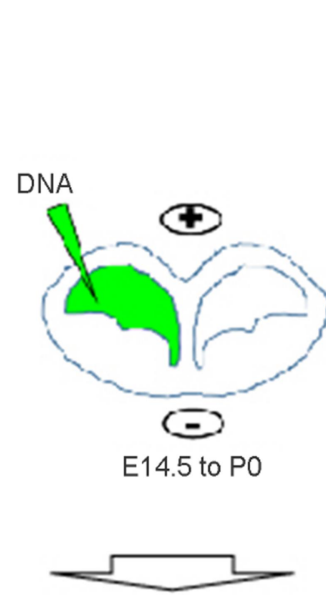

Microarrary
B

Hierarchical clustering

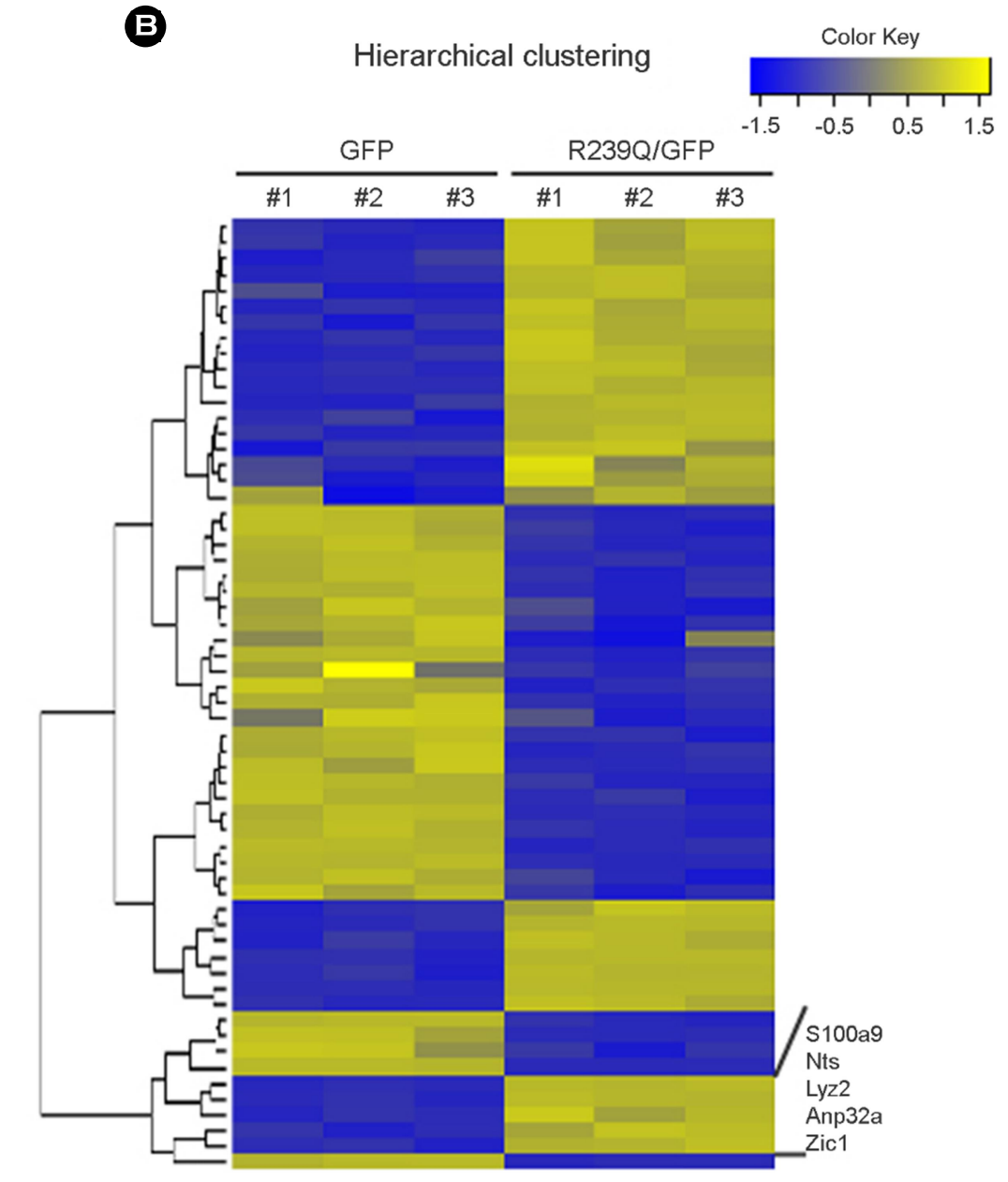

Fig. 3. Microarray analysis of CCN3-R239Q-overexpressing cortical neurons. (A) Schematic illustrating the approach for preparing the total RNA from CCN3-R239Q-overexpressing cortical tissue for microarray. GFP-positive cortical tissues were dissected from three independent $G f p$ and $G f p / C C N 3-\mathrm{R} 239 \mathrm{Q}$ electroporated mouse brains at P0. (B) Hierarchical clustering of 60 genes highly regulated by CCN3R239Q overexpression. Among these, five apoptotic-related genes (S100a9, Nts, Lyz2, Anp32a, and Zicl) showed at least a 1.5-fold upregulation in the CCN3-R239Q-overexpressing cortical tissues. Yellow and blue indicate relatively higher and lower expression, respectively. 


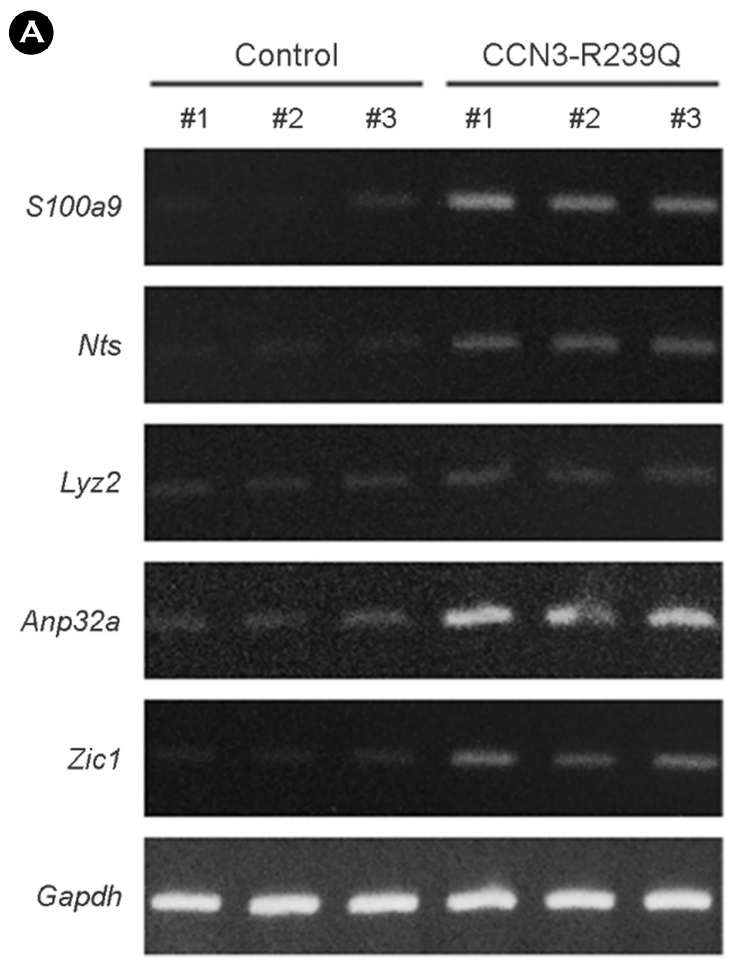

B

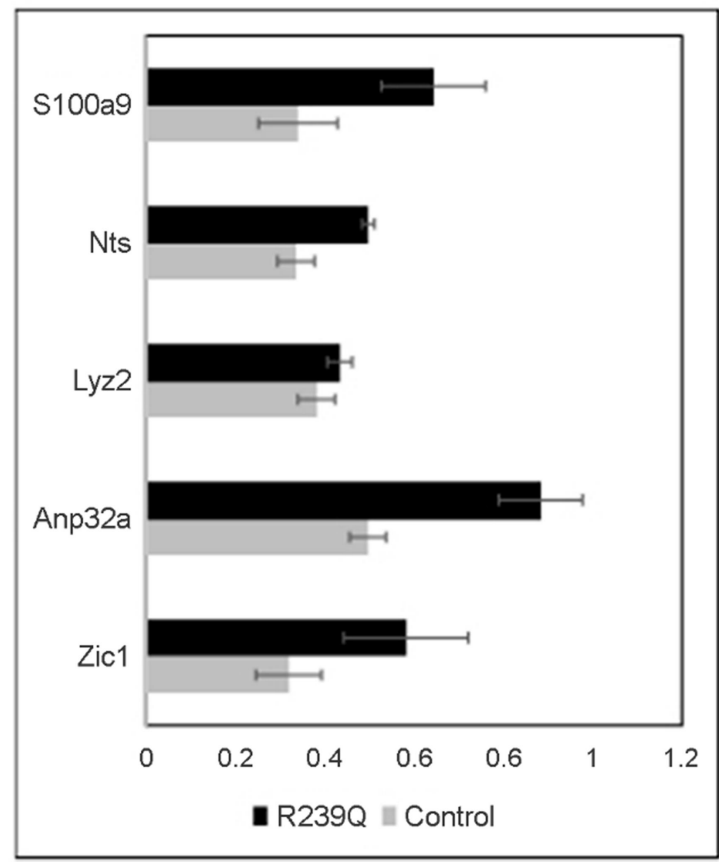

Fig. 4. Apoptotic-related genes upregulated by CCN3-R239Q expression. Microarray analysis showed that CCN3-R239Q upregulated genes related to apoptosis. (A) CCN3-R239Q overexpression increased the expression of S100a9, Nts, Lyz2, Anp32a, and Zicl. Three independent results are shown. CCN3-R239Q forced expression was confirmed by qPCR. (B) Quantitative analysis of the band intensity of qPCR in (A). Results are presented as means \pm standard deviation.

and 67 genes decreased more than 1.5 fold (Supplementary Table 3). We identified that five apoptotic-related genes (S100a9, Nts, Lyz2, Anp32a, and Zic1) were upregulated by CCN3-R239Q expression through hierarchical clustering of the top 60 most abundant variants (30 genes with high upregulation and 30 genes with highly specific downregulation by CCN3-R239Q expression) (Fig. 3B).

To verify whether these identified genes were truly upregulated, qPCR was used to confirm changes in expression of the same total RNA which was used in the microarray. The expression of acidic (leucine-rich) nuclear phosphoprotein 32 family, member A (Anp32a) was increased approximately 2-fold in CCN3-R239Q-overexpressing cortical tissue (Fig. 4). Previous studies have shown that ANP32A has several functions involved in transcriptional regulation, cell survival, and apoptosis (Wang et al., 2015). In particular, this expression pattern was observed in various regions of the brain including the mouse cerebral cortex and cerebellum
(Wang et al., 2015). The temporal expression pattern is evident in early developmental stages. The dynamic pattern decreases until birth, but increases following birth.

Collectively, these results demonstrate that the mutation of Arg239 to glutamine in CCN3 in the mouse cortex from early developmental stages to adulthood can induce abnormal accumulation and aggregation of $\mathrm{CCN} 3$, eventually leading to neuronal cell death by the ectopic expression of apoptotic genes such as Anp32a.

\section{DISCUSSION}

The function of CCN3, which is expressed in the cerebral cortex continuously throughout development and in adulthood (Kang et al., 2011; Park et al., 2015), is still unclear. Previous studies have shown that abnormalities in CCN3 expression can affect neuronal differentiation (Park et al., 2015). It has also recently been shown that the palmitoylation 
of CCN3 is crucial for its extracellular secretion (Kim et al., 2018). Taken together, these results suggest that normal expression and secretion of $\mathrm{CCN} 3$ are important for neuronal differentiation and maintenance of function. Therefore, aberrant $\mathrm{CCN} 3$ expression and secretion are likely to affect neuronal function and/or survival.

$\mathrm{CCN} 3$ is a member of the CCN family with four functional domains and is known to play an important role in various diseases such as injury repair, fibrotic disease, and cancer; as well as cardiovascular development, skeletal development, and neural development (Holbourn et al., 2008; Heath et al., 2008; Chen et al., 2009; Ouellet et al., 2011; Jun and Lau, 2011; Ren et al., 2014; Park et al., 2015; Malik et al., 2015; Fong et al., 2017; Kim et al., 2018). In particular, $\mathrm{CCN1}, 2$, and 3 induce apoptotic cell death in fibroblasts, but prevent apoptosis in endothelial cells (Chen et al., 2009; Jun and Lau, 2011). A previous study elucidated how the receptors and extracellular ligands bind each domain of the CCN protein in this process (Jun and Lau, 2011). Several other studies have reported that $\mathrm{CCN}$ proteins are translocated into the nuclei inside the cells independent of their secretion outside the cell, and may therefore be involved in transcriptional regulation (Jun and Lau, 2011; Fong et al., 2017). Nevertheless, studies on the intracellular function of $\mathrm{CCN}$ proteins are limited. Although there are no reports on the SNPs of CCN proteins associated with certain diseases, it is necessary to investigate the effect of $\mathrm{CCN} 3$ mutations on the cells.

In this study, we focused on CCN3, the most abundantly expressed variant in the cerebral cortex during cortical development. We demonstrated the effect of CCN3 single nucleotide mutations on cells (Fig. 1C, 2). The TSP-1 domain of $\mathrm{CCN} 3$ plays an important role in the extracellular secretion of CCN3 [10], and the CCN3-R239Q mutation induces abnormal intracellular aggregation of $\mathrm{CCN} 3$ as well as extracellular secretion (Fig. 1B). The abnormal intracellular aggregation of $\mathrm{CCN} 3$ induces the expression of apoptotic genes and neuronal degeneration in vivo (Fig. 1C, 3). Overexpression of the CCN3-R239Q mutation, in particular, has been shown to increase the expression of apoptotic-related genes, such as Anp32a, in the developing mouse brain (Fig. 4).

Taken together, the precise expression and secretion of
CCN3 seems to be crucial not only in cerebral development but also in the adult. In particular, the fact that a single mutation of CCN3 shown in this study could induce aberrant apoptosis of neuronal cells strongly suggests that abnormal expression of CCN3 may contribute to the pathogenesis of neurodegenerative diseases.

\section{ACKNOWLEDGEMENTS}

This research was supported by the Brain Research Program through the National Research Foundation of Korea (NRF) funded by the Ministry of Science and ICT (NRF2017M3C7A1046154, 2016R1D1A3B03936255).

\section{CONFLICT OF INTEREST}

The authors have no conflicts of interest to disclose.

\section{REFERENCES}

Brigstock DR. The connective tissue growth factor/cysteine-rich 61/nephroblastoma overexpressed (CCN) family. Endocrine Reviews. 1999. 20: 189-206.

Chen CC, Lau LF. Functions and mechanisms of action of CCN matricellular proteins. The International Journal of Biochemistry \& Cell Biology. 2009. 41: 771-783.

de Oliveira GA, Rangel LP, Costa DC, Silva JL. Misfolding, Aggregation, and Disordered Segments in c-Abl and p53 in Human Cancer. Frontiers in Oncology. 2015. 5: 97.

Fong KW, Zhao JC, Kim J, Li S, Yang YA, Song B, Rittie L, Hu M, Yang X, Perbal B, Yu J. Polycomb-Mediated Disruption of an Androgen Receptor Feedback Loop Drives CastrationResistant Prostate Cancer. Cancer Research. 2017. 77: 412-422.

Heath E, Tahri D, Andermarcher E, Schofield P, Fleming S, Boulter CA. Abnormal skeletal and cardiac development, cardiomyopathy, muscle atrophy and cataracts in mice with a targeted disruption of the Nov (Ccn3) gene. BMC Developmental Biology. 2008. 8: 18.

Holbourn KP, Acharya KR, Perbal B. The CCN family of proteins: structure-function relationships. Trends in Biochemical Sciences. 2008. 33: 461-473.

Jun JI, Lau LF. Taking aim at the extracellular matrix: CCN proteins as emerging therapeutic targets. Nature Reviews Drug Discovery. 2011. 10: 945-963.

Kang HJ, Kawasawa YI, Cheng F, Zhu Y, Xu X, Li M, Sousa AM, 
Pletikos M, Meyer KA, Sedmak G, Guennel T, Shin Y, Johnson MB, Krsnik Z, Mayer S, Fertuzinhos S, Umlauf S, Lisgo SN, Vortmeyer A, Weinberger DR, Mane S, Hyde TM, Huttner A, Reimers M, Kleinman JE, Sestan N. Spatio-temporal transcriptome of the human brain. Nature. 2011. 478: 483-489.

Kim Y, Yang H, Min JK, Park YJ, Jeong SH, Jang SW, Shim S. CCN3 secretion is regulated by palmitoylation via ZDHHC22. Biochemical and Biophysical Research Communications. 2018. 495: 2573-2578.

Lek M, Karczewski KJ, Minikel EV, Samocha KE, Banks E, Fennell T, O'Donnell-Luria AH, Ware JS, Hill AJ, Cummings BB, Tukiainen T, Birnbaum DP, Kosmicki JA, Duncan LE, Estrada K, Zhao F, Zou J, Pierce-Hoffman E, Berghout J, Cooper DN, Deflaux N, DePristo M, Do R, Flannick J, Fromer M, Gauthier L, Goldstein J, Gupta N, Howrigan D, Kiezun A, Kurki MI, Moonshine AL, Natarajan P, Orozco L, Peloso GM, Poplin R, Rivas MA, Ruano-Rubio V, Rose SA, Ruderfer DM, Shakir K, Stenson PD, Stevens C, Thomas BP, Tiao G, TusieLuna MT, Weisburd B, Won HH, Yu D, Altshuler DM, Ardissino D, Boehnke M, Danesh J, Donnelly S, Elosua R, Florez JC, Gabriel SB, Getz G, Glatt SJ, Hultman CM, Kathiresan S, Laakso M, McCarroll S, McCarthy MI, McGovern D, McPherson R, Neale BM, Palotie A, Purcell SM, Saleheen D, Scharf JM, Sklar P, Sullivan PF, Tuomilehto J, Tsuang MT, Watkins HC, Wilson JG, Daly MJ, MacArthur DG. Exome Aggregation Consortium, Analysis of proteincoding genetic variation in 60,706 humans. Nature. 2016. 536: 285-291.

Malik AR, Liszewska E, Jaworski J. Matricellular proteins of the Cyr61/CTGF/NOV (CCN) family and the nervous system. Frontiers in Cellular Neuroscience. 2015. 9: 237.
Ouellet V, Tiedemann K, Mourskaia A, Fong JE, Tran-Thanh D, Amir E, Clemons M, Perbal B, Komarova SV, Siegel PM. CCN3 impairs osteoblast and stimulates osteoclast differentiation to favor breast cancer metastasis to bone. The American Journal of Pathology. 2011. 178: 2377-2388.

Park M, Baek IJ, Kim H, Woo DK, Park YJ, Shim S. CCN3 overexpression inhibits growth of callosal projections via upregulation of RAB25. Biochemical and Biophysical Research Communications. 2015. 461: 456-462.

Perbal B. Alternative splicing of CCN mRNAs it has been upon us. Journal of Cell Communication and Signaling. 2009. 3: 153-157.

Ren Z, Hou Y, Ma S, Tao Y, Li J, Cao H, Ji L. Effects of CCN3 on fibroblast proliferation, apoptosis and extracellular matrix production. International Journal of Molecular Medicine. 2014. 33: 1607-1612

Wang S, Wang Y, Lu Q, Liu X, Wang F, Ma X, Cui C, Shi C, Li J, Zhang D. The expression and distributions of ANP32A in the developing brain. BioMed Research International. 2015. 2015: 207347.

Zhang Y, Wang C. Nephroblastoma overexpressed (NOV/CCN3) gene: a paired-domain-specific PAX3-FKHR transcription target that promotes survival and motility in alveolar rhabdomyosarcoma cells. Oncogene. 2011. 30: 3549-3562.

https://doi.org/10.15616/BSL.2018.24.2.64

Cite this article as: $\mathrm{Kim} \mathrm{H}$, Yang $\mathrm{H}$, Woo DK, Jang SW, Shim S. A Missense Variant (R239Q) in CCN3 Induces Aberrant Apoptosis in the Developing Mouse Brain. Biomedical Science Letters. 2018. 24: 64-75. 